\title{
The Implementation of Wireless Scale Based on the Bluetooth 4.0 Low-energy
}

\author{
Qiao Huang ${ }^{1, a}$, Kejia Chen ${ }^{2, b}$ \\ ${ }^{1}$ Research Institute of Electronic Science and Technology of UESTC, Chengdu. China \\ ${ }^{2}$ Ningbo Research Institute of UESTC, Ningbo. China \\ aemail: hqfriend@126.com, bemail:pursue_pm@foxmail.com
}

Keywords: Bluetooth 4.0; CC2540; Cellphone application; algorithm of automatic identify

\begin{abstract}
Google released the Android 4.3 operating system on July 25, 2013.It ends the situation of manufacturers' alone definition to Bluetooth 4.0, and supports low-energy technology. With the help of integrated chip CC2540 and mobile application, a low-power and smart Bluetooth scale is realized. Using the compression effect of resistance, it produces variational voltage signal. After AD sampling, those signals are sent to cellphone application which owns Bluetooth 4.0 function. When cellphone receives those data, it inserts them into the database. Since it supports multiple users, and owns function of automatic identification for the users. Through successful user login, selecting the authentication, reading data from the database, the system has two display modes, which are tendency chart and list view. What's more, it can point out user' condition of Body Mass Index based on the recent data.
\end{abstract}

\section{Introduction}

There only exists single scales or standalone application for weight management. Even if there are some products which own both scale and cellphone application, but they are mostly based on Bluetooth 3.0.As we know, Bluetooth 3.0 has many advantages ,compared with 2.0,such as higher transmission rate, less exceptions when transmitting data. While in comparison with 4.0, it has a higher cost, cross manufacturers. On July 18, 2013, Le Xin released the first scale, supporting Bluetooth 4.0, which was called A3-BT and allowed to store and display for multi-users. For the reasons of high cost and not apply to android system, the product did not win a big market. To make more people share the Bluetooth 4.0, new smart scale is designed, applying to android system. Combine traditional scale's sensor circuit and CC2540 chip, the scale disposes data via CC2540 and send to cellphone. The application can store weight data and display according to user option. Therefore, it needs a kind of algorithm to automatic identification of information. Organization of the Text

\section{Working Principle}

\section{A.Scale working principle}

With the people's gravity on the scale, it makes the pressure sensors produce deformation and change their shapes. Then the driving voltage start to change, outputting variational analog signals. Usually these signals are very weak, and need to be amplified via amplified filter circuit, outputting to A/D converter later. The CPU receives and processes digital signals, and then send to LED to display, meanwhile, send to CC2540 to store and emit outside via Bluetooth 4.0 

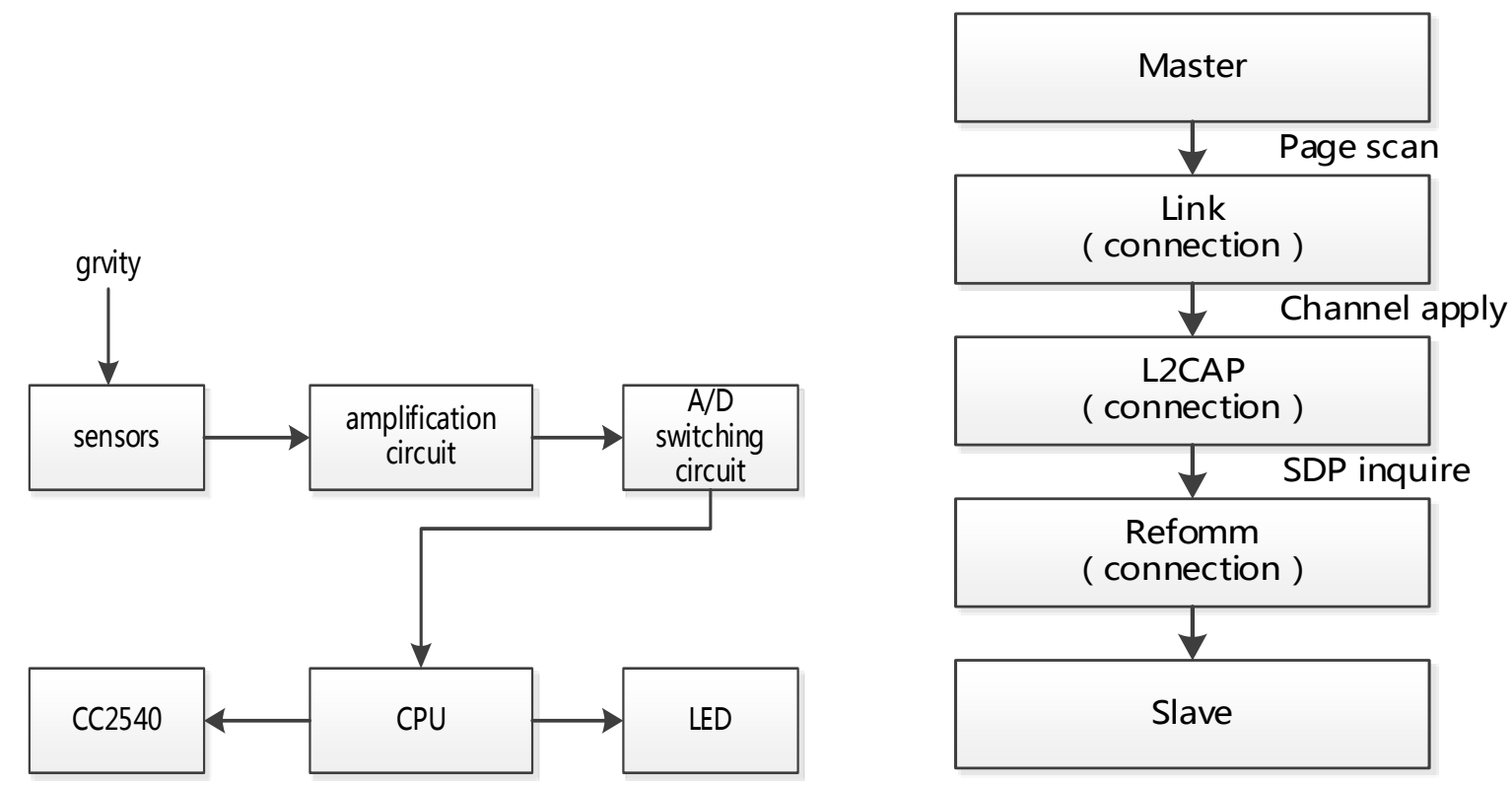

Figure 1. Scale operating principle

Figure 2. The process of Bluetooth connectivity

\section{B.Bluetooth 4.0}

The Bluetooth SIG completed the Bluetooth Core Specification version 4.0 (called Bluetooth Smart) and has been adopted as of 30 June 2010.which has many advantages, such as, fast connecting, low-power, deep sleep. Besides, it adds some new features, which improve consumer usability with increased co-existence support for LTE, bulk data exchange rates, and aid developer innovation by allowing devices to support multiple roles Simultaneously. The process of Bluetooth connection consists of four parts. Through link connection, L2CPA connection, refomm connection, it completes the application connection from master to slave.

\section{Hard System Design}

\section{A. System Chart}

Bluetooth smart scale has four parts, power control module, data processing module, Bluetooth 4.0 module, cellphone application, see figure 3.

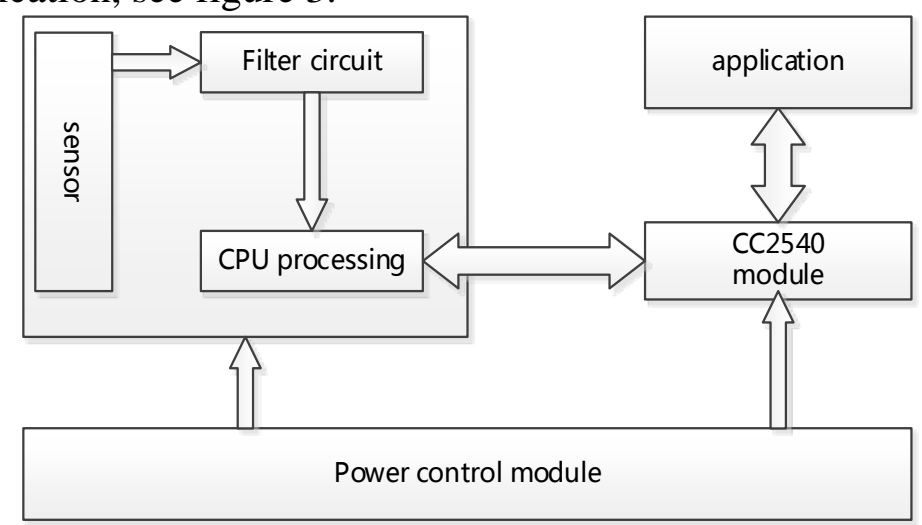

Figure 3. The system chart

\section{B. CC2540 Introduction}

Bluetooth Low Energy (BLE) is a new low-power Bluetooth transmission standard of SIG, saving power more than from 2 to 100 times, the maximum current being the half, compared with the traditional one. On November, 2010, TI designed wireless transceiver chip - CC2540 according to this standard, which was capsulated in a $6 \mathrm{~mm} * 6 \mathrm{~mm}$ integrated controller, reducing its physical size sharply, improving the flexibility design for developers, and especially upgrading easily.

The Bluetooth 4.0 BLE(CC2540) module is cost-effective, low-power module for Bluetooth low energy application, which is suitable for systems where very low power consumption is required, very apply to medical consumption device, Mobil attachment, sporting and healthy application, 
which usually need short transition times between operating modes further enable low power consumption.

\section{Software System Design}

\section{A. MCU Control Program}

Under the help of enhanced 8051 core from CC1540, the MCU control program completes two functions, sampling voltage signal from sensors, via some preprocessing and storing, another is when receiving the connecting and emit instruct from cellphone, it will send out these data, see figure 4 .

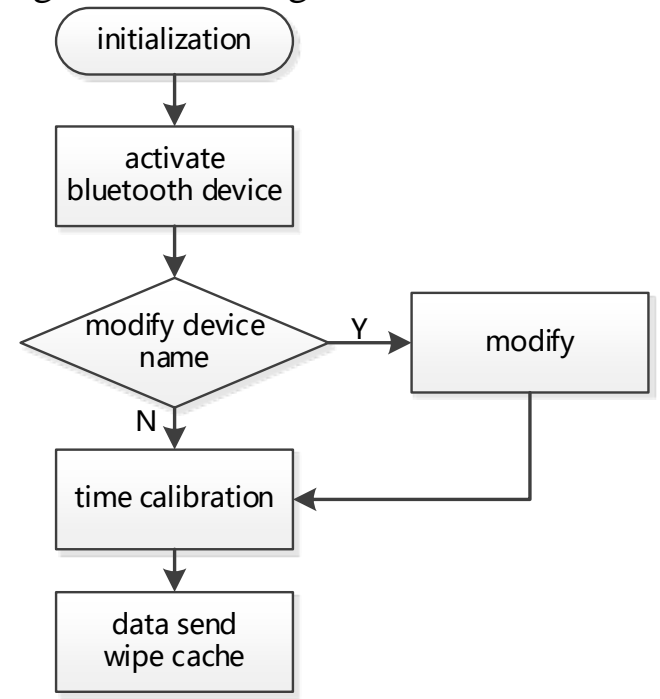

Figure 4. MCU control program

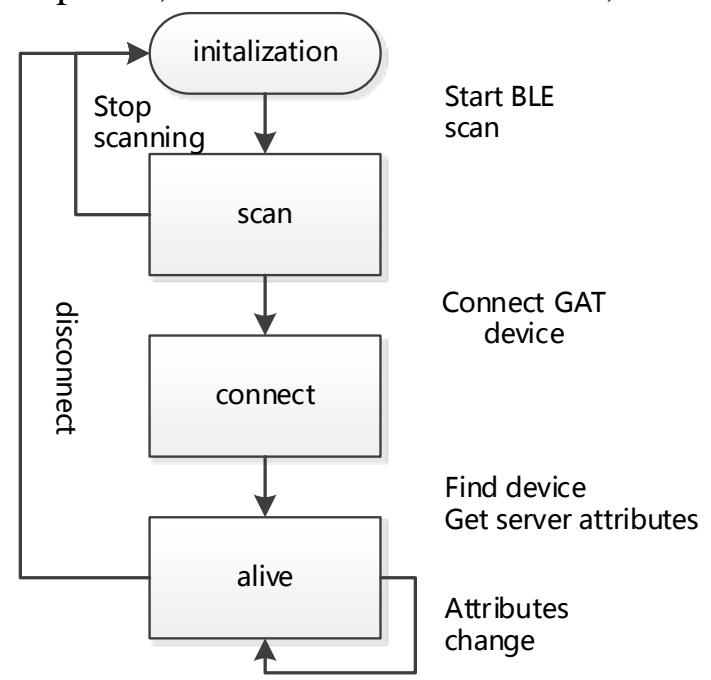

Figure 5. BLE working flow chart

\section{B. Cellphone Application}

The application is made up of three parts, Bluetooth interface layer, data processing layer, user interface layer. Of the three parts, the Bluetooth layer is critical, whose working flow goes through BLE initialization, searching for BLE equipment, connecting, transmitting data, and then removing connection, closing program, see figure 5.Data processing layer gets signal from Bluetooth layer, and store into database via particular disposing. The user interface layer is for interaction, which can display weight data tendency chart, data list in detail and the tips for BMI. What's more, it can be shared to Sina microblog or Friend circle. If the application is the first time to be started, users will see the guide, and tips for upgrade and turn on the Bluetooth switch afterwards. When coming to application's main interface, users will get a tip for scanning equipment before it hasn't connected any before, and once it connects to one successful, it will keeps its address into the database, for automatic connection if the equipment is alive, see the figure 6. 

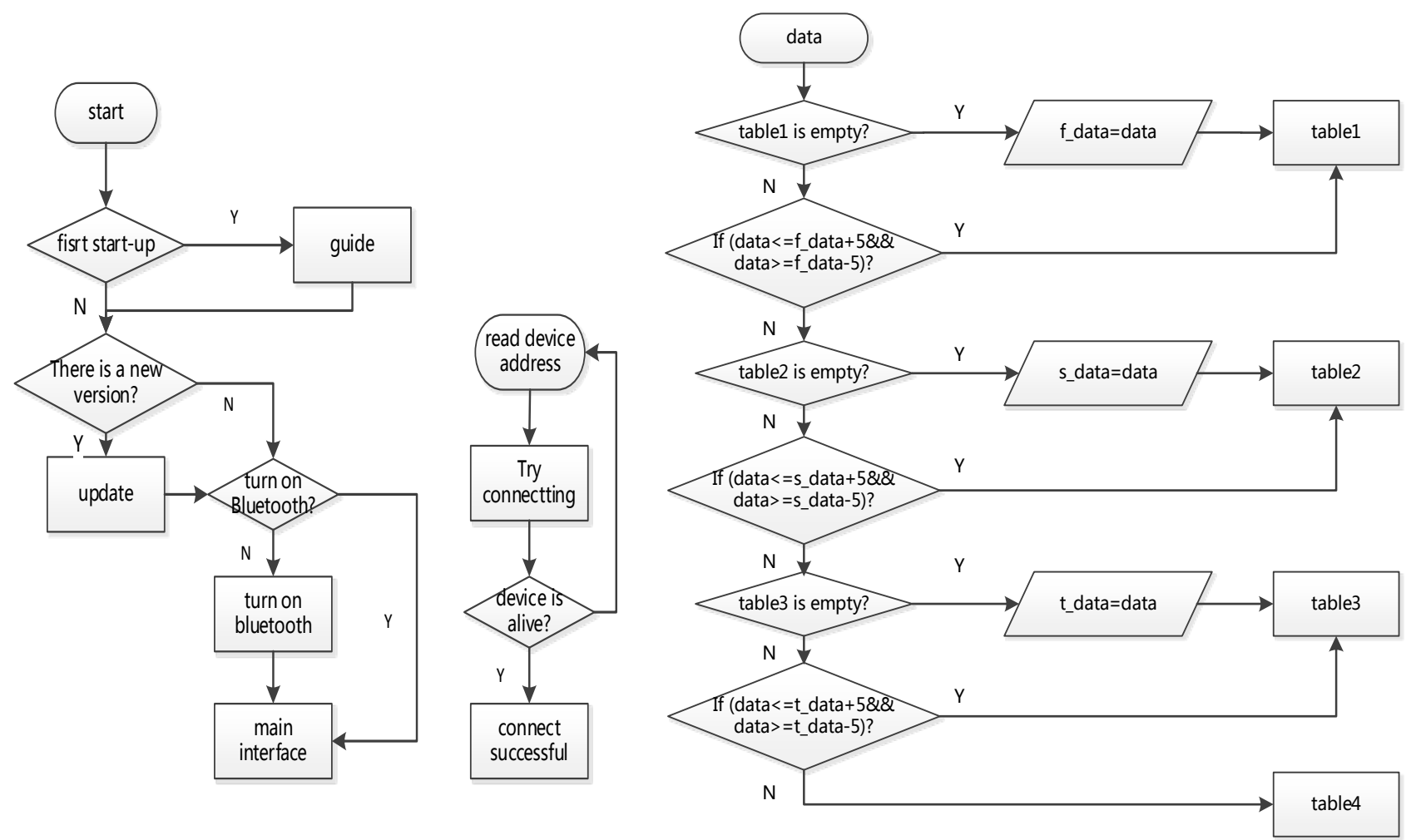

Figure 6. Application working flow chart

Figure 7. The algorithm of automatic identify user

\section{The algorithm of automatic identify user}

For better user experience, the Bluetooth scale activate when people stand on, avoiding any push buttons. Since the cellphone supports multi-users 'data storage and display, the recognition of each user's information becomes very critical. If one scale gets similar data from two or more people, it is impossible to identify. While based on a usual situation, a family consists of three people, and they have obvious difference about weight. According to above analysis, it can figure out a simple and easy algorithm. It can assume an adult people will have weight change less than $5 \mathrm{~kg}$, while the child will have a great change, so the database which changes drastic belongs to the growing child.

\section{Test results}

This system concludes hardware design and cellphone application program, of which hardware is available for data processing, and software is for recording data and display.

Collecting some data from Bluetooth scale, show in table 1, and it can display in the cellphone correctly.

Table 1 testing data

\begin{tabular}{|c|c|}
\hline Testing time & data $(\mathrm{kg})$ \\
\hline 04/06/2014 10:20 am & 62.1 \\
\hline 04/06/2014 21:30 pm & 61.2 \\
\hline 05/06/2014 08:10 am & 62.5 \\
\hline 06/06/2014 08:30 am & 60.5 \\
\hline 07/06/2014 10:10 am & 61.7 \\
\hline $08 / 06 / 201412: 20 \mathrm{am}$ & 63.2 \\
\hline $09 / 06 / 201415: 30 \mathrm{pm}$ & 61.4 \\
\hline $10 / 06 / 201417: 20 \mathrm{pm}$ & 60.4 \\
\hline $13 / 06 / 201409: 00 \mathrm{am}$ & 61.3 \\
\hline $14 / 06 / 201411: 00 \mathrm{am}$ & 59.3 \\
\hline $15 / 06 / 201420: 30 \mathrm{pm}$ & 59.4 \\
\hline $16 / 06 / 201414: 20 \mathrm{pm}$ & 61.1 \\
\hline
\end{tabular}




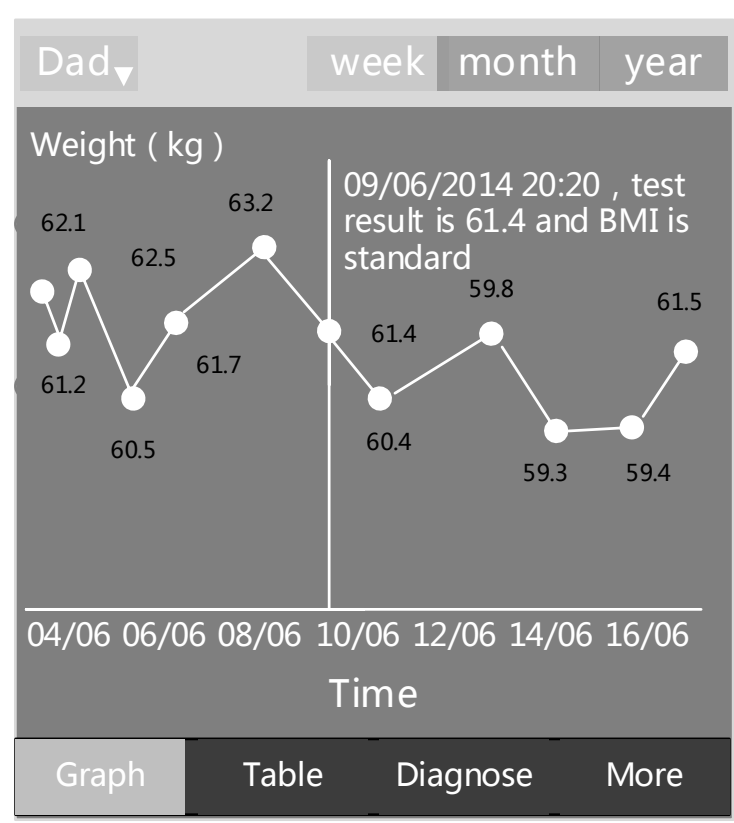

Figure 8. Application chart

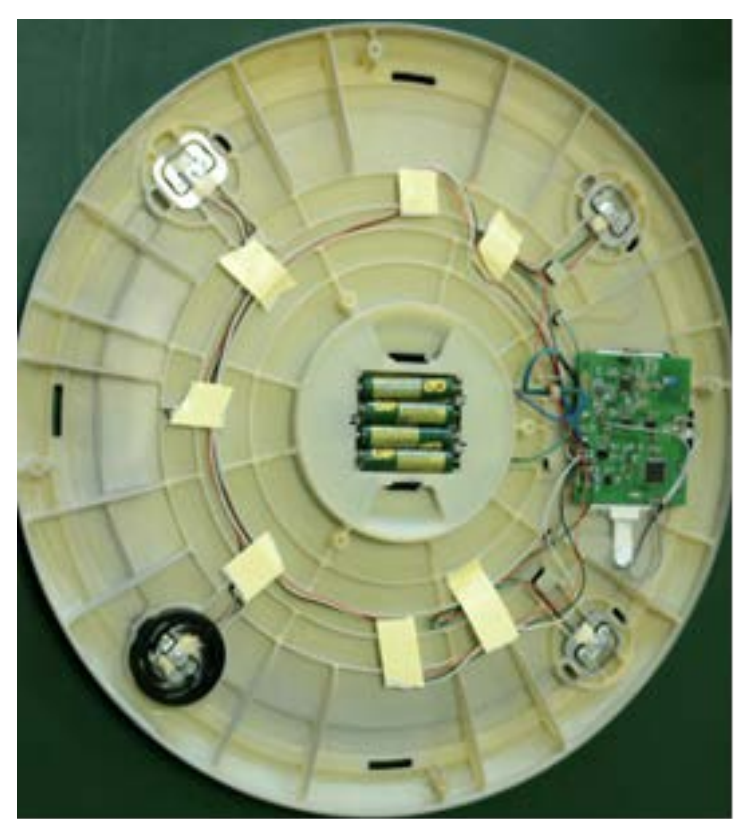

Figure 9. Bluetooth scale chart

\section{Conclusion}

Wireless Bluetooth scale combines BLE and traditional electronic equipment, through scale measuring weight,BLE technology transmitting data to cellphone, and help people to acquaint their weight's change, make a further exercise plan. Due to low-power, low cost of BLE technology, it is widely used in the fields of health care, exercise fitness, home entertainment, and security safeguard. Especially on health care, people will upload body temperature, blood pressure to server via Bluetooth, connecting to clinic or hospital for receiving immediate and effective treatment in case of some exceptions. With the coming of Age of Big Data, the humans get predict their healthy change and get prepared for some major disease in advance under the help of data digging technology.

\section{References}

[1]Wang Wei.Wireless Fish Systemon Bluetooth 4.0low-energy [J]. Piezoelectrics\& Acoustooptics. 2013

[2] TexasInstruments CC2540 Datasheet [EB/OL](2013-6-24).http://www.ti.com/product/cc2540

[3] Fan Chencan.The Android Smartphone’s ECG Monitoring System Based on Bluetooth 4.0 [M] .Zhejiang University 2013

[4] LUJAN S, SUARDIAZ-MURO J, CABRERA-LOZOYA A,et al.Deeploc:discreet indoor people location application. 4th IFIP International Conference on New Technologies, Mobility and Security. 2011

[5] Gao Bo,GE Ning.Design And Implementation of Bluetooth low-energy Consumption Personal Health Network[J].Transducer and Microsystem Technologies. 2013/32/6

[6] Xu Jingou.Principles and Implementation of Energy Bluetooth 4.0 Protocol[J].Microcomputer Application.2012/28/10 